\title{
Gravitational energy loss in high energy particle collisions: ultrarelativistic plunge into a multidimensional black hole
}

\author{
Emanuele Bert:* \\ Department of Physics, Aristotle University of Thessaloniki, Thessaloniki 54124, Greece and \\ McDonnell Center for the Space Sciences, Department of Physics, \\ Washington University, St. Louis, Missouri 63130, USA ${ }^{\dagger}$ \\ Marco Cavagliât \\ Institute of Cosmology and Gravitation, University of Portsmouth, Portsmouth PO1 2EG, UK and \\ Department of Physics and Astronomy, The University of Mississippi, \\ PO Box 1848, University, Mississippi 38677-1848, USA \\ Leonardo Gualtier $\$$ \\ Dipartimento di fisica "G. Marconi", Università di Roma "La Sapienza" \\ and Sezione INFN Roma 1, piazzale Aldo Moro 2, I-00185 Roma, Italy
}

(Dated: November 2, 2018)

\begin{abstract}
We investigate the gravitational energy emission of an ultrarelativistic particle radially falling into a $D$-dimensional black hole. We numerically integrate the equations describing black hole gravitational perturbations and obtain energy spectra, total energy and angular distribution of the emitted gravitational radiation. The black hole quasinormal modes for scalar, vector, and tensor perturbations are computed in the WKB approximation. We discuss our results in the context of black hole production at the $\mathrm{TeV}$ scale.

PACS numbers: 04.70.Bw, 04.50.+h
\end{abstract}

\section{INTRODUCTION}

Brane-world models describe the visible universe as a four-dimensional brane embedded in a higher-dimensional bulk 1]. A generic consequence of the brane-world scenario is that the fundamental gravitational scale is lower than the observed Planck scale. In some models, the fundamental scale is lowered to values that would be accessible to nextgeneration particle colliders, thus enabling laboratory-based studies of strong gravitational physics via perturbative 2] and nonperturbative events [3]. Ultrahigh energy cosmic rays could also probe trans-Planckian energies [4]. The possibility that strong gravitational effects such as black hole $(\mathrm{BH})$ and brane formation could be observed in the near future has sparked a lot of interest in the investigation of nonperturbative gravitational phenomena in hard-scattering events [5]. (For a review and more references, see Ref. [6]).

Trans-Planckian BH formation at energy scales much larger than the fundamental gravitational scale is a classical process [3]. The event is dominated by the $s$-channel and the initial state is modelled by two classical shock waves with given impact parameter. In this context, a major issue is the estimate of the collisional energy loss. The hoop conjecture states that the collision of two particles $i j$ with center-of-mass (c.m.) energy $E_{\mathrm{cm}}$ and impact parameter smaller than the Schwarzschild radius $r_{s}\left(E_{\mathrm{cm}}\right)$ forms a trapped surface [7]. This event is formally described by the process $i j \rightarrow \mathrm{BH}+E(X)$, where $E(X)$ denotes the collisional "junk" energy which does not contribute to the BH mass. The junk energy includes a bulk component of gravitational radiation and other possible non-standard model gauge fields, and a brane component of standard model collisional by-products carrying the charge of the initial particles. The newly formed $\mathrm{BH}$ is expected to decay first by loss of gauge radiation into the bulk and then by thermal Hawking emission. The Hawking evaporation ends when the mass of the $\mathrm{BH}$ approaches the fundamental gravitational scale. At this stage the $\mathrm{BH}$ either decays completely by emitting the residual Planckian energy or leaves a stable remnant with mass about the Planck mass [8]. Most of the observable signatures of BH formation come from Hawking's phase

\footnotetext{
$\dagger$ Present address: Groupe de Cosmologie et Gravitation (GR $\epsilon$ CO), Institut d'Astrophysique de Paris (CNRS), 98 ${ }^{\text {bis }}$ Boulevard Arago, 75014 Paris, France

*Email: berti@astro.auth.gr

‡Email: cavaglia@phy.olemiss.edu

$\S$ Email: leonardo.gualtieri@roma1.infn.it
} 
and strongly depend on the initial BH mass [9]. Hence, a precise calculation of the collisional energy loss is essential to the phenomenology of $\mathrm{BH}$ formation.

A numerical estimate of the total collisional energy loss for spherically symmetric BHs in $D \geq 4$ dimensions has been given by Yoshino and Nambu (YN) [10] (see also Ref. [1]). The YN approach evaluates the total junk energy $E(X)$ by investigating the formation of the $\mathrm{BH}$ apparent horizon [12]. The colliding particles are assumed massless, uncharged and pointlike. Each particle is modelled by an infinitely boosted Schwarzschild solution with fixed energy. This solution describes a plane-fronted gravitational shock wave corresponding to the Lorentz-contracted longitudinal gravitational field (Aichelburg-Sexl wave) [13. The collision is simulated by combining two shock waves travelling in opposite directions. The apparent horizon arises in the union of the two shock waves. The junk energy is estimated by comparing the initial c.m. energy to the BH mass. The result is that the collisional energy loss depends on the impact parameter and increases as the number of spacetime dimensions increases.

The YN method allows estimation of the total junk energy in the classical uncharged point-particle approximation. However, it cannot discriminate between different components of $E(X)$, which is theoretically and experimentally most important. In a realistic BH event such as a proton-proton collision at LHC 14], the BH is formed by the collision of two partons. The bulk component of the junk energy is dominated by gravitational radiation and is invisible to the detector. The gravitational junk energy and the invisible component of Hawking emission (neutrinos, gravitons ...) add to the total missing energy of the process. Therefore, the knowledge of collisional energy loss in gravitational emission should provide a good estimate of the different sources of energy loss and missing energy.

An accurate estimate of the gravitational collisional energy loss would require the use of the full non-linear Einstein equations in $D$ dimensions. This is a formidable task, even in four dimensions. Recently, significant advances in numerical relativity allowed stable numerical simulations of $\mathrm{BH}-\mathrm{BH}$ collisions for initial $\mathrm{BH}$ separation of a few Schwarzschild radii in the non-linear Einstein theory. The gravitational waveforms predicted by these simulations are in excellent agreement with analytical results from first and second order perturbation theory [15]. Since the linearization of the Einstein equations yields results which are surprisingly close to the full theory (see, e.g., Ref. [16]), $\mathrm{BH}$ perturbation theory is likely to provide accurate estimates of gravitational wave emission in higher-dimensional spacetimes. Relying on this result, we compute the gravitational wave emission in higher dimensions via a perturbative approach. Our computation is the first of this kind to our knowledge.

The formalism for the computation of gravitational wave emission from perturbed BHs was developed by Regge and Wheeler [17] and Zerilli [18], who reduced the problem to the solution of two Schrödinger-like equations. Davis et al. [19] computed the energy radiated in the radial infall of a particle of mass $m_{0}$ starting from rest at infinity into a four-dimensional $\mathrm{BH}$ of mass $M_{\mathrm{BH}} \gg m_{0}$. This study was later generalized to the radial infall of a particle with finite initial velocity or starting at finite distance from the $\mathrm{BH}$ 20]. (For a more comprehensive introduction to BH perturbation theory see, e.g., Refs. 21]). Cardoso and Lemos [22, 23] have recently investigated the plunge of ultrarelativistic test particles into a four-dimensional static BH and along the rotation axis of a Kerr BH, improving early estimates by Smarr [24]. In this paper we generalize these results to higher dimensions by computing the gravitational radiation emitted by an ultrarelativistic particle falling into a $D$-dimensional spherically symmetric BH. Since wave propagation in odd-dimensional curved spacetimes is not yet fully understood, we restrict our investigation to even dimensions. (Wave late-time behavior and propagation are very different in odd- and even-dimensional spacetimes 25, 26, 27]. Moreover, open issues in the definition of asymptotic flatness 28] do not allow an unambiguous definition of "gravitational waves radiated at infinity" in odd dimensions.)

We model the particle collision as a relativistic test particle plunging into a $\mathrm{BH}$ with mass $M_{\mathrm{BH}}=E_{\mathrm{cm}}$. We use recent results of $D$-dimensional gravitational-wave theory by Cardoso et al. [25] and the $D$-dimensional extension of Zerilli's formalism by Kodama and Ishibashi (KI) 29, 30, 31, 32], which reduces the problem to the solution of three Schrödinger-like equations. Our method provides a simple and relativistically consistent estimate of the collisional gravitational emission in higher dimensions. We derive the emitted energy in terms of the wave amplitude and study the angular dependence of the radiation using the KI formalism. We also present a systematic calculation of $\mathrm{BH}$ quasinormal modes (QNMs) for the different perturbations in the WKB approach, extending recent calculations by Konoplya [33, 34]. We show that there is a significant relation between the QNM frequencies and the spectral content of the emitted radiation.

The outline of the paper is as follows. In Sec. 2 we introduce our notations and the basic equations. In Sec. 3 ] we briefly describe our numerical approach to the computation of gravitational wave emission (details are in the Appendices). Section 4 contains the main results of the paper. Conclusions are presented in Sec. 5 .

\section{PERTURBATION EQUATIONS AND QUASINORMAL MODES}

In the next subsection we introduce the background metric and the KI perturbation equations [30]. In Subsec. 2.2] we describe the method to compute the BH QNMs. 


\subsection{Background metric and perturbation equations}

The spherically symmetric BH in $D=n+2$ dimensions is described by the Schwarzschild-Tangherlini metric 35]

$$
d s^{2}=-f(r) d t^{2}+\frac{d r^{2}}{f(r)}+r^{2} d \Omega_{n}^{2},
$$

where $d \Omega_{n}$ is the metric of the $n$-dimensional unit sphere $S^{n}$, and

$$
f(r)=1-\frac{2 M}{r^{n-1}}
$$

The $\mathrm{BH}$ mass $M_{\mathrm{BH}}$ is given in terms of the parameter $M$ by

$$
M_{\mathrm{BH}}=\frac{n M \mathcal{A}_{n}}{8 \pi c^{2} G_{n+2}},
$$

where $\mathcal{A}_{n}=2 \pi^{(n+1) / 2} / \Gamma[(n+1) / 2]$ is the area of $S^{n}, G_{n+2}$ is the $(n+2)$-dimensional Newton constant, and $c$ is the speed of light. We will set $G_{n+2}=1$ and $c=1$ in the following. The $(n+2)$-dimensional tortoise coordinate $r_{*}$ is defined by

$$
\frac{d r_{*}}{d r}=\frac{1}{f(r)}
$$

Integrating Eq. (4) we find

$$
r_{*}=r+\frac{2 M}{n-1} \sum_{j=0}^{n-2} \frac{\ln \left(r / \alpha_{j}-1\right)}{\alpha_{j}^{n-2}}
$$

where

$$
\alpha_{j}=(2 M)^{1 /(n-1)} \mathrm{e}^{2 \pi \mathrm{i} j /(n-1)}, \quad(j=0, \ldots, n-2),
$$

and the integration constant has been chosen to make the argument of the logarithm dimensionless. Here and throughout the paper we use the notations of Refs. 29, 30]; the indices $(\mu, \nu),(i, j)$, and $(a, b)$ denote the coordinates of the $D$-dimensional spacetime, the coordinates of $S^{n}$, and the coordinates of the two-dimensional spacetime $(t, r)$, respectively.

Kodama and Ishibashi [30] showed that the gravitational perturbation equations for this metric can be reduced to Schrödinger-like wave equations:

$$
\left(\frac{d^{2}}{d r_{*}^{2}}+\omega^{2}-V\right) \Phi=0,
$$

where the potential $V$ depends on the kind of perturbation. Setting $x \equiv 2 M / r^{n-1}$, the potential for scalar perturbations is

$$
V_{S}=\frac{f(r) Q(r)}{16 r^{2} H^{2}}
$$

where

$$
\kappa^{2}=l(l+n-1), \quad l=0,1,2, \ldots \quad m=\kappa^{2}-n, \quad H(r)=m+\frac{n(n+1)}{2} x,
$$

and

$$
\begin{aligned}
Q(r) & =n^{4}(n+1)^{2} x^{3}+n(n+1)\left[4\left(2 n^{2}-3 n+4\right) m+n(n-2)(n-4)(n+1)\right] x^{2} \\
& -12 n[(n-4) m+n(n+1)(n-2)] m x+16 m^{3}+4 n(n+2) m^{2}
\end{aligned}
$$

Equation (8) reduces to the Zerilli equation [18] for $n=2$. The potential for vector perturbations is

$$
V_{V}=\frac{f}{r^{2}}\left[\kappa_{V}^{2}+1+\frac{n(n-2)}{4}-\frac{3 n^{2} M}{2 r^{n-1}}\right]
$$


where

$$
\kappa_{V}^{2}=l(l+n-1)-1, \quad l=1,2, \ldots
$$

Equation (11) reduces to the Regge-Wheeler equation [17] for $n=2$. Finally, the potential for tensor perturbations is

$$
V_{T}=\frac{f}{r^{2}}\left[\kappa_{T}^{2}+2+\frac{n(n-2)}{4}+\frac{n^{2} M}{2 r^{n-1}}\right]
$$

where

$$
\kappa_{T}^{2}=l(l+n-1)-2, \quad l=1,2, \ldots
$$

Equation (13) was derived by Gibbons and Hartnoll [36] in a more general case (see also [37], where a Gauss-Bonnet term is included) and has no equivalent in four dimensions.

\subsection{Quasinormal modes}

The knowledge of the QNM frequencies of multidimensional BHs enables a clear physical interpretation of their gravitational emission. QNMs are free damped BH oscillations which are characterized by pure ingoing radiation at the BH horizon and pure outgoing radiation at infinity. The no-hair theorem implies that QNM frequencies depend only on the BH mass, charge and angular momentum. Numerical simulations of $\mathrm{BH}$ collapse and $\mathrm{BH}-\mathrm{BH}$ collision show that, after a transient phase depending on the details of the process, the newly formed BH has a ringdown phase, i.e., it undergoes damped oscillations that can be described as a superposition of slowly damped QNMs (modes with small imaginary part). Furthermore, the QNMs determine the late-time evolution of perturbation fields in the BH exterior (for comprehensive reviews on QNMs see Refs. 38]).

Gravitational radiation from four-dimensional astrophysical BHs is dominated by slowly damped modes. In the following we show that these also dominate the emission of gravitational radiation in higher dimensions and determine important properties of the energy spectra. Recently, Konoplya computed slowly damped QNMs of higher-dimensional BHs 33, 34] using the WKB method. This method is known to be inaccurate for large imaginary parts, but it is accurate enough for the slowly damped modes which are relevant in our context. Therefore, QNM frequencies for scalar, vector, and tensor gravitational perturbations are computed here in the WKB approximation. Our results are in good agreement with those presented by Konoplya in Ref. 34] (modulo a different normalization). At variance with Ref. [34], we concentrated on uncharged black holes in asymptotically flat, even dimensional spacetimes. We extended Konoplya's calculation in two ways: i) in addition to the fundamental QNM we also computed the first two overtones; ii) we carried out our calculations for a much larger range of values of $l$ (Ref. [34] only shows results for $l=2$ and $l=3$ ).

The method consists in applying the WKB approximation to the potential in Eq. (7) with appropriate boundary conditions. The result is a pair of connection formulae which relate the amplitudes of the waves on either side of the potential barrier, and ultimately yield an analytical formula for the QNM frequencies (for details see Refs. 39, 40]). The WKB QNM frequencies $\omega^{2}$ are given in terms of the potential maximum $V_{0}$ and of the potential derivatives at the maximum by

$$
\omega^{2}=\left(V_{0}+\sqrt{-2 V_{0}^{\prime \prime}} \Lambda\right)-i\left(j+\frac{1}{2}\right) \sqrt{-2 V_{0}^{\prime \prime}}(1+\Omega), \quad j=0,1,2, \ldots
$$

where

$$
\begin{aligned}
\Lambda=\frac{1}{\sqrt{-2 V_{0}^{\prime \prime}}} & {\left[\frac{1}{8} \frac{V_{0}^{(4)}}{V_{0}^{\prime \prime}}\left(\frac{1}{4}+\alpha^{2}\right)-\frac{1}{288}\left(\frac{V_{0}^{\prime \prime \prime}}{V_{0}^{\prime \prime}}\right)^{2}\left(7+60 \alpha^{2}\right)\right], } \\
\Omega=\frac{1}{\sqrt{-2 V_{0}^{\prime \prime}}} & {\left[\frac{5}{6912}\left(\frac{V_{0}^{\prime \prime \prime}}{V_{0}^{\prime \prime}}\right)\left(77+188 \alpha^{2}\right)-\frac{1}{384}\left(\frac{V_{0}^{\prime \prime \prime} V_{0}^{(4)}}{V_{0}^{\prime \prime 3}}\right)\left(51+100 \alpha^{2}\right)+\right.} \\
& \left.+\frac{1}{2304}\left(\frac{V_{0}^{(4)}}{V_{0}^{\prime \prime}}\right)^{2}\left(67+68 \alpha^{2}\right)+\frac{1}{288}\left(\frac{V_{0}^{\prime \prime \prime} V_{0}^{(5)}}{V_{0}^{\prime \prime 2}}\right)\left(19+28 \alpha^{2}\right)-\frac{1}{288}\left(\frac{V_{0}^{(6)}}{V_{0}^{\prime \prime}}\right)\left(5+4 \alpha^{2}\right)\right],
\end{aligned}
$$


$\alpha=j+1 / 2$ and $j$ is the mode index. The QNM frequencies for the scalar, vector, and tensor potentials of Sec. 2 and various dimensions are shown in Tables [IV and will be discussed in Sec. 4 Let us stress that the application of the WKB technique is questionable in a few higher-dimensional cases; for $l=2$ and $l=3$ the vector and scalar potentials in $D>6$ are not positive definite and/or display a second, small scattering peak close to the BH horizon. An accurate analysis of these potentials would require a refinement of the standard WKB technique, which is not presented here. These special cases are denoted with italic numbers in Tables III IV

We mention that highly damped QNMs of four- and higher-dimensional BHs have recently become a subject of great interest in a different context. A few years ago, Hod proposed to use Bohr's correspondence principle to determine the $\mathrm{BH}$ area quantum from highly damped BH QNMs 41]. Hod's proposal is quite general: the asymptotic QNM frequency for scalar perturbations of a non-rotating BH in $D$ dimensions is the same as in four dimensions 42 . Quite notably, this result holds also for scalar, vector and tensor gravitational perturbations [42, 43]. Ref. [44] contains a partial list of references on recent developments in this field.

\section{INTEGRATION METHOD}

The computation of the gravitational wave emission of an ultrarelativistic particle plunging into a $\mathrm{BH}$ requires the numerical integration of the inhomogeneous wave equation for scalar gravitational perturbations. (Vector and tensor gravitational perturbations are not excited by a particle in radial infall.) The source term $S^{(n)}$ for the corresponding wave equation in $n+2$ dimensions can be calculated from the stress-energy tensor of the infalling particle. Details of the derivation are in Appendix A

The integration in $(n+2)$ dimensions proceeds as in four dimensions [19, 20]. A good summary of the integration procedure can be found in Ref. 22]. In this section we simply stress the differences between the four- and the $(n+2)$-dimensional cases. For the sake of simplicity, in our numerical integrations we set the horizon radius $r_{h}=$ $(2 M)^{1 /(n-1)}=1$. The equation for the scalar perturbations is

$$
\left(\frac{d^{2}}{d r_{*}^{2}}+\omega^{2}-V_{S}\right) \Phi=S^{(n)}
$$

The general solution of Eq. (18) is obtained via a Green function technique as follows. Consider two independent (left and right) solutions of the homogeneous equation with boundary conditions $\Phi_{L} \sim e^{-\mathrm{i} \omega r_{*}}$ for $r_{*} \rightarrow-\infty$, and $\Phi_{R} \sim e^{\mathrm{i} \omega r_{*}}$ for $r_{*} \rightarrow+\infty$. For $r_{*} \rightarrow+\infty$ the left solution is a superposition of ingoing and outgoing waves of the form

$$
\Phi_{L} \sim B(\omega) e^{\mathrm{i} \omega r_{*}}+C(\omega) e^{-\mathrm{i} \omega r_{*}}
$$

The Wronskian is given by $W=2 \mathrm{i} \omega C(\omega)$. The wave amplitude is obtained from a convolution of the left solution with the source term

$$
\Phi=\frac{1}{W} \int_{-\infty}^{+\infty} \Phi_{L} S^{(n)} d r_{*}
$$

The energy spectrum can be expressed in terms of the wave amplitude as (details of the derivation are given in Appendix [B]

$$
\frac{d E}{d \omega}=\frac{\omega^{2}}{16 \pi} \frac{n-1}{n} \kappa^{2}\left(\kappa^{2}-n\right)|\Phi|^{2}
$$

where $\kappa^{2} \equiv l(l+n-1)$. The Wronskian for a given value of $\omega$ is obtained by integrating the homogeneous equation from a point located as close as possible to the horizon, and expanding $\Phi_{L}$ as

$$
\Phi_{L} \sim e^{-i \omega r_{*}}\left[1+a_{n+2}(r-1)+\ldots\right]
$$

where

$$
a_{n+2}=\frac{-\left(l^{4}+2 l^{3}-l^{2}-2 l+3\right)+(n-2)\left[-2 l^{3}+l^{2}+\left(n^{2}+1\right) l-\left(n^{3}+4 n^{2}+n+6\right) / 4\right]}{(2 \mathrm{i} \omega-1)\left(l^{2}+l+1\right)+(n-2)\left[-l^{2}+(2 \mathrm{i} \omega-n) l+(n+1) \mathrm{i} \omega-\left(n^{2}+1\right) / 2\right]} .
$$

$C(\omega)$ (and $W$ ) can be obtained with good accuracy by matching the numerically integrated $\Phi_{L}$ to the asymptotic expansion

$$
\Phi_{L} \sim e^{\mathrm{i} \omega r_{*}}\left[1+\frac{a_{n+2}(\omega)}{r}+\frac{b_{n+2}(\omega)}{r^{2}}+\ldots\right]+e^{-\mathrm{i} \omega r_{*}}\left[1+\frac{a_{n+2}(-\omega)}{r}+\frac{b_{n+2}(-\omega)}{r^{2}}+\ldots\right]
$$


where the leading-order coefficient is

$$
a_{n+2}(\omega)=\frac{\mathrm{i}\left[l^{2}+(n-1) l+n(n-2) / 4\right]}{2 \omega} .
$$

For given $n, l$ and $\omega$, the error on the Wronskian and on the energy spectrum is typically of the order of $O\left(10^{-4}\right)$.

\section{RESULTS}

The main results of our work are the computation of the QNM frequencies in the WKB approximation, the computation of the energy spectra, and the estimate of the total energy and angular distribution of the radiation emitted during the plunge. These results are discussed in detail below.

\subsection{Quasinormal frequencies}

The WKB QNM frequencies for different even values of $n$ are listed in Tables IIV Each line shows the first three quasinormal frequencies $(j=0,1,2)$ for scalar, vector, and tensor perturbations at given $l$. For $n=2$ tensor perturbations do not exist. In this case the scalar and vector entries correspond to the QNMs of the Zerilli and Regge-Wheeler equations, which are known to be isospectral [21]. The isospectrality is broken for $n>2$. This has been shown analytically by Kodama and Ishibashi [30] and later verified numerically by Konoplya [34]. The real and imaginary parts of scalar QNM frequencies at given $n, l$ and $j$ are smaller than those of vector QNMs, which are in turn smaller than those of tensor QNMs. Since scalar modes are the least damped, they are likely to dominate the gravitational radiation emission.

As $l$ grows, the isospectrality tends to be restored. In the eikonal limit $l \rightarrow \infty$ the centrifugal term of the potential dominates and is the same for scalar, vector, and tensor perturbations. In this limit, the QNM frequencies for all perturbations are

$$
\omega_{R} \sim \frac{n+2 l-1}{2}\left(\frac{2}{n+1}\right)^{\frac{1}{n-1}}\left(\frac{n-1}{n+1}\right)^{1 / 2}, \quad \omega_{I} \sim \frac{n-1}{2(n+1)^{1 / 2}}\left(\frac{2}{n+1}\right)^{\frac{1}{n-1}}(2 j+1) .
$$

The previous relation was derived in Ref. 33] for multidimensional BH perturbations induced by a scalar field. (Notice that the normalization used in Ref. [33] is different from ours.) Here we have shown that it also holds for gravitational perturbations. Isospectrality of scalar and gravitational perturbations is a common feature of the eikonal limit and of the large-damping limit [42, 43] for any $n$.

\subsection{Multipolar components of the energy spectra}

The numerical integration described in Sec. 3 gives the energy spectra of Figs. 1 and 2 The spectra for $n=2$ (top left panel in Fig. (1) are in excellent agreement with those of Ref. [22]. The spectra are flat in the region between the zero-frequency limit and a "cutoff" frequency $\omega_{c}$, beyond which they fall exponentially to zero. The cutoff frequency $\omega_{c}$ is given by the fundamental QNM frequency to a good level of accuracy. This result can be understood in terms of gravitational-wave scattering from the potential barrier which surrounds the black hole. $\omega^{2}$ plays the role of the energy in the Schrödinger-like equation (7). From Eq. (15) it follows that $\omega^{2}=V_{0}$ at first order in the WKB approximation. Therefore, only the radiation with energy smaller than the peak of the potential is backscattered to infinity; radiation with larger frequency is exponentially suppressed.

The gravitational emission of a two-particle hard collision in higher dimensions has been computed by Cardoso et al. 25] using techniques developed in four dimensions by Weinberg [45] and later used by Smarr 24]. The main result of Ref. 25] is that the spectra in $n+2$ dimensions grow as $\omega^{n-2}$, thus the integrated spectra diverge as $\omega^{n-1}$. Physically meaningful results for the total energy can only be obtained by imposing some cutoff on the integrated spectra. Smarr 24] first suggested to use the inverse horizon radius as a cutoff. The relativistic perturbative calculation in $n=2$ [22] shows that the cutoff frequency at fixed $l$ is very close to the fundamental BH QNM. Therefore, the cutoff frequency should be given by some "weighted average" of the fundamental gravitational QNM frequencies 25].

Our results for the spectra and the QNMs confirm the above picture. Fig. 1 shows that all spectra go to zero as $\omega \rightarrow 0$. For $\omega<\omega_{c}$ the spectrum at fixed $l$ is

$$
\frac{d E_{l}}{d \omega}=f_{n, l} \omega^{n-2},
$$


where $f_{n, l}$ is a constant that can be found by a fit of the spectra. For large $l f_{n, l}$ decays as

$$
f_{n, l}=k_{n+2} l^{-3(n+2) / 4} .
$$

A fit of the numerical data gives $k_{4}=2.25, k_{6}=0.832, k_{8}=0.184$, and $k_{10}=0.040$. Our result for $n=2$ is consistent with that of Ref. [22].

As conjectured in Ref. 25], all spectra have a maximum at some cutoff frequency $\omega_{c}$. This cutoff frequency is very close to the fundamental QNM frequency $\omega_{l n}$ for (scalar) gravitational perturbations with given $l$ and $n$, which is marked by open circles in Fig. 1 and Fig. 2 The deviation between $\omega_{c}$ and $\omega_{l n}$ is of order $10 \%$ for low $l$, and decreases for large $l$ (compare Fig. [1] and Fig. [2 to the first column of Tables [IV). The deviation is larger when the WKB method is least reliable, namely for $l=2$ and $n>4$. In these cases, the location of the peaks in the spectra can presumably be used as a more reliable estimate of the QNM frequency. The spectrum decays exponentially for $\omega>\omega_{c}$ with an $n$-dependent slope $\alpha_{n+2}$ (see Fig. 2):

$$
\frac{d E_{l}}{d \omega} \sim e^{-\alpha_{n+2}\left(\omega-\omega_{c}\right)} .
$$

Thus the $\omega$-integrated multipolar contributions $\Delta E_{l}$ at given $l$ are finite. With our choice of units, Cardoso and Lemos [22] find $\alpha_{4}=13.5 \alpha$ (here $\alpha$ is a constant of order unity which cannot easily be determined because the spectra decay very quickly). Our numerical fits give $\alpha_{4} \simeq 15$, in good agreement with their result. In higher dimensions the constants $\alpha_{n+2}$ are comparatively easier to determine. Their values are $\alpha_{6} \simeq 5.5, \alpha_{8} \simeq 3.4$, and $\alpha_{10} \simeq 2.3$. It is not clear if there is any relation between this $n$-dependent slope and the late-time tail behavior predicted in Ref. [26].

Fig. 1 shows that higher multipoles contribute more as $n$ grows. This is evident when we look at the $\omega$-integrated multipolar components of the energy spectra of Fig. 3. The quadrupole $(l=2)$ is dominant only for $n=2$ and $n=4$. For $n=6$ and $n=8$ the dominant multipoles are $l=4$ and $l=6$, respectively (see Table $\nabla$ ). This effect may be related to the appearance of a negative well in the scalar potentials for $l=2$ and $n>4$. It would be interesting to understand better the physical relation between the dominant multipole and the spacetime dimension.

\subsection{Total energy}

The total emitted energy is obtained by numerically integrating the results of the previous section over $\omega$ and summing the multipolar components. For large $l$ the integrated energy in the multipole $l$ can be fitted by

$$
\Delta E_{l}=a_{n+2} l^{-b_{n+2}},
$$

where $\left(a_{6}=0.110, b_{6}=1.69\right),\left(a_{8}=0.050, b_{8}=1.64\right)$, and $\left(a_{10}=0.022, b_{10}=1.40\right)$ for $n=4, n=6$, and $n=8$, respectively. The coefficients $\left(a_{n+2}, b_{n+2}\right)$ have been obtained by fitting the data from $l=14$ to $l=20$ and are weakly dependent on the chosen range of $l$. This variability affects our final results on the total energy within less than a few percent. is

Restoring the dependence on the $\mathrm{BH}$ horizon $r_{h}$ and on the conserved particle energy $p_{0}$, the total emitted energy

$$
E_{\mathrm{em}}=\frac{p_{0}^{2}}{M_{\mathrm{BH}}} \frac{n \mathcal{A}_{n}}{16 \pi} \sum_{l=2}^{\infty} \Delta E_{l} \equiv \frac{p_{0}^{2}}{M_{\mathrm{BH}}} \frac{n \mathcal{A}_{n}}{16 \pi} E_{\mathrm{tot}}^{(D)} \equiv \frac{p_{0}^{2}}{M_{\mathrm{BH}}} \mathcal{E}_{\mathrm{tot}}^{(D)}
$$

where $E_{\mathrm{tot}}^{(D)}$ is the "dimensionless" total energy (expressed in the units $r_{h}=1$ that we used in our numerical integrations). We obtained the integrated spectra numerically up to $l=20$ and extrapolated them for larger $l$ using the fits in Eq. (30). Results are presented in Table VI

Following Ref. 22] we estimate the gravitational energy loss for a collision of two particles with equal mass $M$ by the replacement $p_{0} \rightarrow M, M_{\mathrm{BH}} \rightarrow M_{\mathrm{tot}}=2 M$. For $n=2$ this extrapolation gives results in good agreement with the perturbative shock-wave calculation of Ref. 12] which considers two BHs of equal mass. An analogous extrapolation for $n=2$ gives results in close agreement with the fully relativistic computation [16] for a particle starting from infinity at rest. Therefore, we believe that our extrapolation should provide a qualitative but realistic estimate. The results for different values of $n$ are given in the last column of Table VI The gravitational energy loss is $\sim 13 \%, \sim 10 \%, 7 \%$, and $8 \%$ for $n=2$ to $n=8$, respectively. The result for $n=2$ is in good agreement with previous estimates [12] (see the discussion in Ref. [22]). 


\subsection{Angular dependence}

The angular dependence of the radiation is obtained by evaluating numerically Eq. (B17) and Eq. (B18). Fig. 4 shows the angular dependence of the total energy up to $l=15$ for $n=2, n=4$ and $n=6$.

The angular distribution of the gravitational radiation in the $\mathrm{BH}$ frame goes to zero along the axis of the collision $(\theta=0, \pi)$ in any dimensions. Therefore, the gravitational emission is never back or forward scattered. In four dimensions the angular spectrum of the gravitational radiation increases rapidly at small $\theta$ and becomes approximately flat at greater angles with a maximum in the direction orthogonal to the axis of the collision, before falling rapidly to zero for values of the angles close to $\pi$. The angular distribution of the gravitational radiation for $n>2$ is peaked at $\pm \theta$ and $\pi \pm \theta$, where $\theta$ is a small angle. The difference between the behavior of the angular distribution in four dimensions and in higher dimensions has no evident physical reason. It would be interesting to further explore this point.

\section{CONCLUSION AND PERSPECTIVES}

In this paper we have computed the gravitational emission of a two-particle collision in an even $D$-dimensional spacetime. We have presented the numerical results for $D=4$ to 10 . The collision has been modelled as a massless test particle plunging into a $\mathrm{BH}$ with mass equal to the c.m. energy of the event.

According to our estimates, the total emitted energy in a head-on collision with particles of equal mass ranges from $\sim 13 \%(D=4)$ to $\sim 8 \%(D=10)$. This shows that the loss in gravitational radiation is quite stable under variation of the spacetime dimension and slightly decreases for higher $D$. The result for $D=4$ confirms previous numerical and analytical calculations 12$]$.

Our result contrasts with the YN estimation for the initial mass of a BH in head-on collisions [10]. A possible explanation is that the junk emission is not wholly gravitational emission. The YN method predicts the mass within the apparent horizon to be $\sim 0.71 E_{\mathrm{cm}}$ in four dimensions. If all the junk energy were gravitational radiation, this would amount to a total loss of around 30\%. The disagreement is likely not due to numerical uncertainties or inaccurate approximations: the YN mass decreases for higher spacetime dimensions $\left(\sim 0.71 E_{\mathrm{cm}}\right.$ to $\sim 0.58 E_{\mathrm{cm}}$ for $D=4$ to $D=11$ ), whereas the loss in gravitational radiation remains stable. Since both YN and our methods are purely gravitational, this "dark component" of the junk radiation should describe the by-products of the collision. According to this picture, $\sim 60 \%$ of the c.m. energy in ten dimensions is trapped inside the horizon, $\sim 10 \%$ is emitted in gravitational radiation, and $\sim 30 \%$ goes into particle by-products in the final state. These could be the carriers of the initial charge in a collision between charged particles. For $D>4$ a fraction of the by-products may be emitted into the bulk.

Let us conclude by briefly discussing the phenomenological consequences of these results for $\mathrm{BH}$ formation at the TeV scale. Although uncertainties may affect the numerical estimates, different approaches now confirm that some of the initial c.m. energy is not trapped inside the BH horizon. For head-on collisions in $D=10$, for example, this junk energy ranges from $\sim 10 \%$ (optimistic value - our result) to $\sim 40 \%$ (pessimistic value - YN result). Hence, the initial mass of the $\mathrm{BH}$ formed in the collision could be considerably smaller than the c.m. energy. The experimental signatures of $\mathrm{BH}$ production at particle colliders and in ultrahigh energy cosmic ray events strongly depend on the initial $\mathrm{BH}$ mass. The total multiplicity of the Hawking phase in ten dimensions could be almost halved in the pessimistic case, leading to a greater average energy of the emitted quanta.

A thorough investigation of the effects of energy loss in TeV-scale BH production is undoubtedly worth pursuing. Future research should focus on the extension of the above results to spacetimes with odd dimensions and to gravitational events with different geometries. BHs produced in colliders, for instance, possess nonvanishing angular momentum. Rotating BHs are expected to lose more energy in gravitational waves than Schwarzschild BHs of equal mass. A larger gravitational emission is also expected for non-spherically symmetric BHs. This is particularly relevant when the compactified space is asymmetric, and some of the extra dimensions have size of order of the fundamental gravitational scale. It would be extremely important to quantify these differences.

\section{Acknowledgments}

We warmly thank V. Cardoso for his encouragement to carry out this work and for several useful suggestions. M. C. is grateful to E.-J. Ahn for many helpful comments on the content of this paper. This work has been supported by the EU Programme 'Improving the Human Research Potential and the Socio-Economic Knowledge Base' (Research Training Network Contract HPRN-CT-2000-00137). M. C. is partially supported by PPARC. 


\section{APPENDIX A: THE SOURCE TERM}

In this Appendix we derive the source term of the KI equation that describes the radial plunge of a massless particle into the $(n+2)$-dimensional BH. The perturbation of the stress-energy tensor is

$$
\delta T^{\mu \nu}=-\frac{p_{0}}{\sqrt{-g}} \int d \lambda \delta^{n}(x-x(\lambda)) \frac{d x^{\mu}}{d \lambda} \frac{d x^{\nu}}{d \lambda},
$$

where $p_{0}$ is the conserved energy of the particle. The only non-vanishing components of the particle velocity are $u^{t}$ and $u^{r}$. Thus the source excites only scalar perturbations. Following the notations of Ref. [29], Eq. (A1) reads

$$
\delta T_{\mu \nu}=\left(\begin{array}{c|c}
\tau_{a b} \mathcal{S} & 0 \\
\hline 0 & 0
\end{array}\right)
$$

where $\mathcal{S}$ are the scalar harmonics and $\tau_{a b}$ are the non-vanishing gauge-invariant perturbations of the stress-energy tensor. The $\mathrm{BH}+$ source system is symmetric under rotation of the $(n-1)$-sphere $S^{n-1}$ [6]. Consequently, the harmonic decomposition of the fields contains only harmonics invariant under $S^{n-1}$. We can write the metric of $S^{n}$ as

$$
d \Omega_{n}\left(\theta, \phi_{1}, \ldots, \phi_{n-1}\right)=d \theta^{2}+\sin ^{2} \theta d \Omega_{n-1}\left(\phi_{1}, \ldots, \phi_{n-1}\right)
$$

and choose the trajectory of the test particle to be $\theta=0$. The harmonics which are invariant under $S^{n-1}$ do not depend on $\phi_{1}, \ldots, \phi_{n}$. The scalar harmonics on $S^{n}$ belong to the representations $D^{(l)}$ of $S O(n+1)$

$$
D^{(l)}=\underbrace{\square \ldots}_{l} \text {. }
$$

Each harmonic is labelled by the index $l$ denoting its representation and by additional indices in the representation. We fix a particular element of each representation $D^{(l)}$ (the singlet under $S O(n-1)$ ) by requiring the harmonics to be invariant under $S^{n-1}$. Therefore, the harmonics in the expansion of the perturbations depend only on $l$ and on the dimension $n$ of the sphere. A $(n+2)$-dimensional scalar field can then be expanded as [46]

$$
\phi\left(t, r, \theta, \phi_{1}, \ldots, \phi_{n-1}\right)=\sum_{l} \tilde{\phi}_{l}(t, r) \mathcal{S}^{(n l)}(\theta),
$$

where $\mathcal{S}^{(n l)}(\theta)$ satisfy

$$
D_{i} D^{i} \mathcal{S}^{(n l)}=-\kappa^{2} \mathcal{S}^{(n l)}, \quad \kappa^{2} \equiv l(l+n-1),
$$

and

$$
\int d \Omega_{n} \mathcal{S}^{(n l)} \mathcal{S}^{*\left(n l^{\prime}\right)}=\delta_{l l^{\prime}}
$$

The solution of Eq. (A6) is

$$
\mathcal{S}^{(n l)}(\theta)=K^{(n l)} C_{l}^{(n-1) / 2}(\theta),
$$

where $C_{l}^{(n-1) / 2}(\theta)$ are Gegenbauer polynomials [47] and $K^{(n l)}$ are normalization factors. Using Eq. (A7) we have

$$
K^{(n l)}=\left[\frac{2^{3-n} \pi^{n / 2+1}}{\Gamma\left(\frac{n}{2}\right)} \frac{\Gamma(l+n-1)}{\left(l+\frac{n}{2}-\frac{1}{2}\right) \Gamma\left(\frac{n}{2}-\frac{1}{2}\right)^{2} \Gamma(l+1)}\right]^{-1 / 2} .
$$

The scalar harmonics for the source are obtained setting $\theta=0$ :

$$
S^{(n l)}(\theta=0)=K^{(n l)} \frac{(l+n-2) !}{(n-2) ! l !} .
$$

For a massive particle in radial geodesic motion

$$
\frac{d t}{d r}=-\frac{1}{f(r)}
$$


From equations (A11) and (4) it follows that

$$
r_{*}(r)=-t(r)
$$

where we have set to zero the integration constant. The stress-energy tensor perturbations are

$$
\begin{aligned}
\delta T_{r r}\left(t, r, \theta, \phi_{1}, \ldots\right) & =-p_{0} f^{-2} r^{-n} \delta(t-t(r)) \delta^{n}\left(\Omega_{n}\right), \\
\delta T_{t r}\left(t, r, \theta, \phi_{1}, \ldots\right) & =-p_{0} f^{-1} r^{-n} \delta(t-t(r)) \delta^{n}\left(\Omega_{n}\right) .
\end{aligned}
$$

$\left(\delta T_{t t}\right.$ does not contribute to the source of the KI equation.) Integrating Eqs. A13) on $S^{n}$ and applying a Fouriertransform, the gauge-invariant perturbations are

$$
\begin{aligned}
& \tau_{r r}(\omega, r)=-p_{0} f^{-2} r^{-n} \frac{e^{\mathrm{i} \omega t(r)}}{\sqrt{2 \pi}} S^{(n l)}(\theta=0), \\
& \tau_{t r}(\omega, r)=-p_{0} f^{-1} r^{-n} \frac{e^{\mathrm{i} \omega t(r)}}{\sqrt{2 \pi}} S^{(n l)}(\theta=0) .
\end{aligned}
$$

The source term $S^{(n)}$ for scalar gravitational perturbations is obtained in terms of $\tau_{a b}$ by substituting Eqs. A14 in Eq. (5.44) of Ref. [32], where

$$
S_{a b}=8 \pi r^{n-2} \tau_{a b}, \quad S_{a}=S_{T}=\mathcal{A}=\tilde{J}_{a}=0
$$

The result is:

$$
\begin{aligned}
S^{(2)} & =e^{-\mathrm{i} \omega r_{*}} \frac{8 \sqrt{4 l+2}}{\mathrm{i} \omega r} \frac{(l-1)(l+2)(r-1)}{[(l+2)(l-1) r+3]^{2}}, \\
S^{(4)} & =e^{-\mathrm{i} \omega r_{*}} \frac{16 \sqrt{(2 l+3) \lambda_{2}}}{\sqrt{2 \pi} \mathrm{i} \omega r^{3}} \frac{\left[\left(l^{2}+3 l\right)\left(r^{6}-r^{3}\right)+5-4 r^{6}-r^{3}\right]}{\left[(l+4)(l-1) r^{3}+10\right]^{2}}, \\
S^{(6)} & =e^{-\mathrm{i} \omega r_{*}} \frac{6 \sqrt{(2 l+5) \lambda_{4}}}{\pi \mathrm{i} \omega r^{4}} \frac{\left[\left(l^{2}+5 l\right)\left(r^{10}-r^{5}\right)+14-6 r^{10}-8 r^{5}\right]}{\left[(l+6)(l-1) r^{5}+21\right]^{2}}, \\
S^{(8)} & =e^{-\mathrm{i} \omega r_{*}} \frac{4 \sqrt{(2 l+7) \lambda_{6}}}{\sqrt{3 \pi^{3}} \mathrm{i} \omega r^{5}} \frac{\left[\left(l^{2}+7 l\right)\left(r^{14}-r^{7}\right)+27-8 r^{14}-19 r^{7}\right]}{\left[(l+8)(l-1) r^{7}+36\right]^{2}},
\end{aligned}
$$

where $\lambda_{k} \equiv[(l+k)(l+k-1) \ldots(l+1)]$. The BH horizon $r_{h}$ and the particle energy $p_{0}$ have been set equal to one. Equation A16 coincides with Eq. (8) of Ref. 22].

\section{APPENDIX B: ENERGY AND ANGULAR DISTRIBUTION}

We derive here the formulae for the energy and the angular dependence of the gravitational emission. Gravitational waves in a $(n+2)$-dimensional spacetime behave asymptotically as $\sim r^{-n / 2}[25]$ and possess $n(n+1) / 2-1$ degrees of freedom. The transverse-traceless (TT) gauge is defined by $\delta g_{a b}=0, \delta g_{a i}=0$, and $\delta g_{i j} \gamma^{i j}=0$. These conditions can be chosen by imposing the harmonic gauge in the wave zone and using the remaining gauge freedom to constrain $\delta g_{t t}=0, \delta g_{t i}=0$, and $\gamma^{i j} \delta g_{i j}=0$.

We separate the angular part of the perturbations using tensor spherical harmonics, following Ref. 30]. In the TTgauge, the only nonvanishing term in the decomposition is the $H_{T}$ component. Hence, the gauge invariant quantities for scalar perturbations $\left(F\right.$ and $\left.F_{a b}\right)$ depend only on $H_{T}$ :

$$
F=\frac{1}{n} H_{T}+\frac{1}{r}\left(D^{a} r\right) X_{a}, \quad F_{a b}=D_{a} X_{b}+D_{b} X_{a},
$$

where

$$
X_{a}=\frac{r^{2}}{\kappa^{2}} D_{a} H_{T}
$$

The scalar perturbation $\Phi$ is written in terms of the gauge invariant quantities as

$$
\Phi=\frac{n \tilde{Z}-r(X+Y)}{r^{n / 2-1}\left[\kappa^{2}-n+n(n+1) x / 2\right]},
$$


where

$$
X+Y=-2 n r^{n-2} F, \quad \tilde{Z}=\frac{1}{\mathrm{i} \omega} r^{n-2} F_{t}^{r} .
$$

Setting

$$
H_{T} \rightarrow \frac{A}{r^{n / 2}} e^{\mathrm{i} \omega r_{*}}
$$

the asymptotic behavior of $\Phi$ is

$$
\lim _{r \rightarrow \infty} \Phi=\frac{2 A}{\kappa^{2}} e^{\mathrm{i} \omega r_{*}} .
$$

The asymptotic behavior of $h_{i j}$ in the TT-gauge is

$$
h_{i j}^{T T}=2 r^{2} H_{T} \mathcal{S}_{i j} \sim \frac{\kappa^{2} \Phi}{r^{n / 2-2}} \mathcal{S}_{i j}=\frac{\Phi}{r^{n / 2-2}}\left(D_{i} D_{j} \mathcal{S}+\frac{\kappa^{2}}{n} \gamma_{i j} \mathcal{S}\right) .
$$

The energy-momentum pseudotensor does not depend on the spacetime dimension and is given by 25]

$$
\frac{d E}{d S d t}=<t^{00}>=\frac{\omega^{2}}{32 \pi}<h_{i j}^{T T} h^{T T i j}>,
$$

where $h_{i j}^{T T}$ are metric perturbations in the time domain. Using Parseval's theorem, the energy-momentum pseudotensor in the frequency domain is

$$
\frac{d E}{d S d \omega}=\frac{\omega^{2}}{32 \pi}<\tilde{h}_{i j}^{T T} \tilde{h}^{* T T i j}>
$$

where $\tilde{h}_{i j}^{T T}$ are now the metric perturbations in the frequency domain. Substituting Eq. (B7) in Eq. (B9) we get

$$
\frac{d E}{d S d \omega}=\frac{\omega^{2}}{32 \pi} \frac{|\Phi|^{2}}{r^{n}}\left(D_{i} D_{j} \mathcal{S}+\frac{\kappa^{2}}{n} \gamma_{i j} \mathcal{S}\right)\left(D_{k} D_{l} \mathcal{S}^{*}+\frac{\kappa^{2}}{n} \gamma_{k l} \mathcal{S}^{*}\right) \gamma^{i k} \gamma^{j l}
$$

Integrating on the sphere $d S=r^{n} d \Omega_{n}$ and using the relations

$$
D_{i} D^{i} \mathcal{S}=-\kappa^{2} \mathcal{S}, \quad\left[D_{j}, D_{i}\right] V^{j}=R_{k i} V^{k}=(n-1) V_{i},
$$

where $V^{i}$ is a generic vector (here and in the following, indices are raised and lowered with $\gamma^{i j}$ ), we find the "two-sided" power spectrum

$$
\frac{d E}{d \omega}_{\text {two-sided }}=\int d S^{(n)} \frac{d E}{d S d \omega}=\frac{\omega^{2}}{32 \pi} \frac{n-1}{n} \kappa^{2}\left(\kappa^{2}-n\right)|\Phi|^{2} .
$$

The "one-sided" spectrum in Eq. (21) is obtained by multiplying Eq. (B12) by two. In the four-dimensional limit the one-sided spectrum is

$$
\left.\frac{d E}{d \omega}\right|_{n=2}=\frac{\omega^{2}}{32 \pi} \frac{(l+2) !}{(l-2) !}|\Phi|^{2} .
$$

Equation (B13) is Zerilli's formula for the $l$-th multipole component of the energy spectrum in four-dimensions. The total energy spectrum is given by the sum over the multipoles.

For each $l$ the energy spectrum is

$$
\frac{d E_{l}}{d \Omega d \omega}=\frac{\omega^{2}}{32 \pi}\left|\Phi_{l}\right|^{2}\left(D_{i} D_{j} \mathcal{S}+\frac{\kappa^{2}}{n} \gamma_{i j} \mathcal{S}\right)\left(D^{i} D^{j} \mathcal{S}^{*}+\frac{\kappa^{2}}{n} \gamma^{i j} \mathcal{S}^{*}\right)
$$

Substituting Eq. (B12) in the previous equation, we find

$$
\frac{d E_{l}}{d \Omega d \omega}=\frac{d E_{l}}{d \omega} \frac{n}{n-1} \frac{1}{\kappa^{2}\left(\kappa^{2}-n\right)}\left(D_{i} D_{j} \mathcal{S}+\frac{\kappa^{2}}{n} \gamma_{i j} \mathcal{S}\right)\left(D^{i} D^{j} \mathcal{S}^{*}+\frac{\kappa^{2}}{n} \gamma^{i j} \mathcal{S}^{*}\right)
$$


The angular dependence for the $l$-th multipole is obtained by integrating over frequency [22]. The result is

$$
\frac{d E_{l}}{d \Omega}=\Delta E_{l} \frac{n}{n-1} \frac{1}{\kappa^{2}\left(\kappa^{2}-n\right)}\left(D_{i} D_{j} \mathcal{S}+\frac{\kappa^{2}}{n} \gamma_{i j} \mathcal{S}\right)\left(D^{i} D^{j} \mathcal{S}^{*}+\frac{\kappa^{2}}{n} \gamma^{i j} \mathcal{S}^{*}\right) \equiv \Delta E_{l} \Lambda_{l}(\theta),
$$

where

$$
\Lambda_{l}(\theta)=\frac{1}{\kappa^{2}\left(\kappa^{2}-n\right)}\left(\frac{n}{n-1} \mathcal{S}_{, \theta \theta}+\frac{k}{n-1} \mathcal{S}\right)^{2} .
$$

For $n=2$ Eq. (B17) reduces to the known result in four dimensions [22]. The angular dependence is obtained by summing over the multipoles:

$$
\frac{d E}{d \Omega}(\theta)=\sum_{l} \frac{d E_{l}}{d \Omega}=\sum_{l} \Delta E_{l} \Lambda_{l}(\theta) .
$$

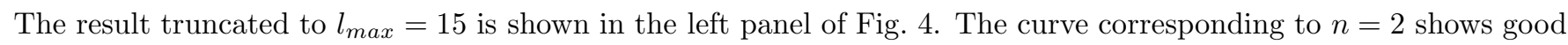
agreement with Fig. 3 of Ref. [22].

[1] I. Antoniadis, Phys. Lett. B 246, 377 (1990); N. Arkani-Hamed, S. Dimopoulos and G. R. Dvali, Phys. Lett. B 429, 263 (1998) arXiv:hep-ph/9803315; I. Antoniadis, N. Arkani-Hamed, S. Dimopoulos and G. R. Dvali, Phys. Lett. B 436, 257 (1998) arXiv:hep-ph/9804398; L. Randall and R. Sundrum, Phys. Rev. Lett. 83, 3370 (1999) arXiv:hep-ph/9905221; L. Randall and R. Sundrum, Phys. Rev. Lett. 83, 4690 (1999) arXiv:hep-th/9906064.

[2] G. F. Giudice, R. Rattazzi and J. D. Wells, Nucl. Phys. B 544, 3 (1999) arXiv:hep-ph/9811291; T. Han, J. D. Lykken and R. J. Zhang, Phys. Rev. D 59, 105006 (1999) arXiv:hep-ph/9811350; J. L. Hewett, Phys. Rev. Lett. 82, 4765 (1999) arXiv:hep-ph/9811356; T. G. Rizzo, Phys. Rev. D 59, 115010 (1999) arXiv:hep-ph/9901209.

[3] T. Banks and W. Fischler, arXiv:hep-th/9906038 S. B. Giddings and S. Thomas, Phys. Rev. D 65, 056010 (2002) arXiv:hep-ph/0106219; S. Dimopoulos and G. Landsberg, Phys. Rev. Lett. 87, 161602 (2001) arXiv:hep-ph/0106295; S. Dimopoulos and R. Emparan, Phys. Lett. B 526, 393 (2002) arXiv:hep-ph/0108060; R. Casadio and B. Harms, Int. J. Mod. Phys. A 17, 4635 (2002) arXiv:hep-th/0110255; E. J. Ahn, M. Cavaglià and A. V. Olinto, Phys. Lett. B 551, 1 (2003) arXiv:hep-th/0201042.

[4] J. L. Feng and A. D. Shapere, Phys. Rev. Lett. 88, 021303 (2002) arXiv:hep-ph/0109106 ; A. Ringwald and H. Tu, Phys. Lett. B 525, 135 (2002) arXiv:hep-ph/0111042; E. J. Ahn, M. Ave, M. Cavaglià and A. V. Olinto, Phys. Rev. D 68, 043004 (2003) arXiv:hep-ph/0306008.

[5] S. Hossenfelder, S. Hofmann, M. Bleicher and H. Stocker, Phys. Rev. D 66, 101502 (2002) arXiv:hep-ph/0109085; K. Cheung, Phys. Rev. Lett. 88, 221602 (2002) arXiv:hep-ph/0110163; S. I. Dutta, M. H. Reno and I. Sarcevic, Phys. Rev. D 66, 033002 (2002) arXiv:hep-ph/0204218; K. Cheung, Phys. Rev. D 66, 036007 (2002) arXiv:hep-ph/0205033; E. J. Ahn and M. Cavaglià, Gen. Rel. Grav. 34, 2037 (2002) arXiv:hep-ph/0205168 .; V. Frolov and D. Stojkovic, Phys. Rev. D 66, 084002 (2002) arXiv:hep-th/0206046; T. Han, G. D. Kribs and B. McElrath, Phys. Rev. Lett. 90, 031601 (2003) arXiv:hep-ph/0207003 ; V. Frolov and D. Stojkovic, Phys. Rev. Lett. 89, 151302 (2002) arXiv:hep-th/0208102; I. Mocioiu, Y. Nara and I. Sarcevic, Phys. Lett. B 557, 87 (2003) arXiv:hep-ph/0301073; A. Chamblin, F. Cooper and G. C. Nayak, arXiv:hep-ph/0301239

[6] M. Cavaglià, Int. J. Mod. Phys. A 18, 1843 (2003) arXiv:hep-ph/0210296.

[7] K.S. Thorne, in: Magic without magic: John Archibald Wheeler, edited by J. Klauder (freeman, San Francisco, 1972).

[8] M. Cavaglià, S. Das and R. Maartens, Class. Quant. Grav. 20, L205 (2003) arXiv:hep-ph/0305223; S. Hossenfelder, M. Bleicher, S. Hofmann, H. Stocker and A. V. Kotwal, arXiv:hep-ph/0302247

[9] M. Cavaglià, Phys. Lett. B 569, 7 (2003) arXiv:hep-ph/0305256 .

[10] H. Yoshino and Y. Nambu, Phys. Rev. D 67, 024009 (2003) arXiv:gr-qc/0209003.

[11] O. I. Vasilenko, arXiv:hep-th/0305067

[12] P. D. D'Eath and P. N. Payne, Phys. Rev. D 46, 658 (1992); P. D. D'Eath and P. N. Payne, Phys. Rev. D 46, 675 (1992); P. D. D'Eath and P. N. Payne, Phys. Rev. D 46, 694 (1992); D. M. Eardley and S. B. Giddings, Phys. Rev. D 66, 044011 (2002) arXiv:gr-qc/0201034; H. Yoshino and Y. Nambu, Phys. Rev. D 66, 065004 (2002) arXiv:gr-qc/0204060; D. Ida and K. I. Nakao, Phys. Rev. D 66, 064026 (2002) arXiv:gr-qc/0204082. O. I. Vasilenko, arXiv:hep-th/0305067

[13] P. C. Aichelburg and R. U. Sexl, Gen. Rel. Grav. 2, 303 (1971).

[14] http://lhc-new-homepage.web.cern.ch/lhc-new-homepage/

[15] R. J. Gleiser, C. O. Nicasio, R. H. Price and J. Pullin, Phys. Rept. 325, 41 (2000) arXiv:gr-qc/9807077.

[16] P. Anninos and S. Brandt, Phys. Rev. Lett. 81, 508 (1998) arXiv:gr-qc/9806031.

[17] T. Regge, J. A. Wheeler, Phys. Rev. 108, 1063 (1957).

[18] F. Zerilli, Phys. Rev. D 2, 2141 (1970).

[19] M. Davis, R. Ruffini, W. H. Press, R. H. Price, Phys. Rev. Lett. 27, 1466 (1971). 
[20] R. Ruffini, Phys. Rev. D 7, 972 (1973); R. Ruffini, V. Ferrari, Phys. Lett. B 98, 381 (1981); C. Lousto, R. H. Price, Phys. Rev. D 55, 2124 (1997).

[21] J. Baker, B. Brugmann, M. Campanelli, C. O. Lousto and R. Takahashi, Phys. Rev. Lett. 87, 121103 (2001) arXiv:gr-qc/0102037; Y. Mino, M. Sasaki, M. Shibata, H. Tagoshi and T. Tanaka, Prog. Theor. Phys. Suppl. 128, 1 (1997) arXiv:gr-qc/9712057; S. Chandrasekhar, The Mathematical Theory of Black Holes, Oxford University Press (1983).

[22] V. Cardoso and J. P. Lemos, Phys. Lett. B 538, 1 (2002) arXiv:gr-qc/0202019.

[23] V. Cardoso and J. P. Lemos, Gen. Rel. Grav. 35, 327 (2003) arXiv:gr-qc/0207009.

[24] L. Smarr, Phys. Rev. D 15, 2069 (1977).

[25] V. Cardoso, O. J. Dias and J. P. Lemos, Phys. Rev. D 67, 064026 (2003) arXiv:hep-th/0212168.

[26] V. Cardoso, S. Yoshida, O. J. Dias and J. P. Lemos, arXiv:hep-th/0307122

[27] A. O. Barvinsky and S. N. Solodukhin, arXiv:hep-th/0307011

[28] S. Hollands and A. Ishibashi, arXiv:gr-qc/0304054

[29] H. Kodama, A. Ishibashi and O. Seto, Phys. Rev. D 62, 064022 (2000) arXiv:hep-th/0004160.

[30] H. Kodama and A. Ishibashi, arXiv:hep-th/0305147

[31] A. Ishibashi and H. Kodama, arXiv:hep-th/0305185

[32] H. Kodama and A. Ishibashi, arXiv:hep-th/0308128

[33] R. A. Konoplya, Phys. Rev. D 68, 024018 (2003) arXiv:gr-qc/0303052.

[34] R. A. Konoplya, Phys. Rev. D 68, 124017 (2003) arXiv:hep-th/0309030.

[35] F. R. Tangherlini, Nuovo Cimento 27, 636 (1963).

[36] G. Gibbons and S. A. Hartnoll, Phys. Rev. D 66, 064024 (2002) arXiv:hep-th/0206202.

[37] I. P. Neupane, arXiv:hep-th/0302132

[38] K. D. Kokkotas and B. G. Schmidt, Living Rev. Rel. 2, 2 (1999) arXiv:gr-qc/9909058; H.-P. Nollert, Class. Quant. Grav. 16, R159 (1999).

[39] S. Iyer and C. M. Will, Phys. Rev. D 35, 3621 (1987).

[40] S. Iyer, Phys. Rev. D 35, 3632 (1987).

[41] S. Hod, Phys. Rev. Lett. 81, 4293 (1998) arXiv:gr-qc/9812002.

[42] L. Motl and A. Neitzke, arXiv:hep-th/0301173

[43] D. Birmingham, arXiv:hep-th/0306004

[44] O. Dreyer, Phys. Rev. Lett. 90, 081301 (2003) arXiv:gr-qc/0211076; G. Kunstatter, Phys. Rev. Lett. 90, 161301 (2003) arXiv:gr-qc/0212014; L. Motl, Adv. Theor. Math. Phys. 6, 1135 (2003) arXiv:gr-qc/0212096; E. Berti and K. D. Kokkotas, Phys. Rev. D 67, 064020 (2003) arXiv:gr-qc/0301052; V. Cardoso and J. P. Lemos, Phys. Rev. D 67, 084020 (2003) arXiv:gr-qc/0301078; C. Molina, Phys. Rev. D 68, 064007 (2003) arXiv:gr-qc/0304053; A. Neitzke, arXiv:hep-th/0304080 A. Maassen van den Brink, Phys. Rev. D 68, 047501 (2003) arXiv:gr-qc/0304092; V. Cardoso, R. Konoplya and J. P. Lemos, Phys. Rev. D 68, 044024 (2003) arXiv:gr-qc/0305037; D. Birmingham, S. Carlip and Y. j. Chen, arXiv:hep-th/0305113 E. Berti, V. Cardoso, K. D. Kokkotas and H. Onozawa, arXiv:hep-th/0307013 N. Andersson and C. J. Howls, arXiv:gr-qc/0307020 S. Hod, arXiv:gr-qc/0307060 S. Yoshida and T. Futamase, arXiv:gr-qc/0308077 S. Musiri and G. Siopsis, arXiv:hep-th/0308168 J. Oppenheim, arXiv:gr-qc/0307089

[45] S. Weinberg, Gravitation and Cosmology (Wiley, New York, 1972).

[46] V. Cardoso and J. P. Lemos, Phys. Rev. D 66, 064006 (2002) arXiv:hep-th/0206084.

[47] M. Abramowitz, I. A. Stegun, in Handbook of Mathematical functions (Dover, New York, 1970); useful information can also be found at http://www.mathworld.wolfram.com 

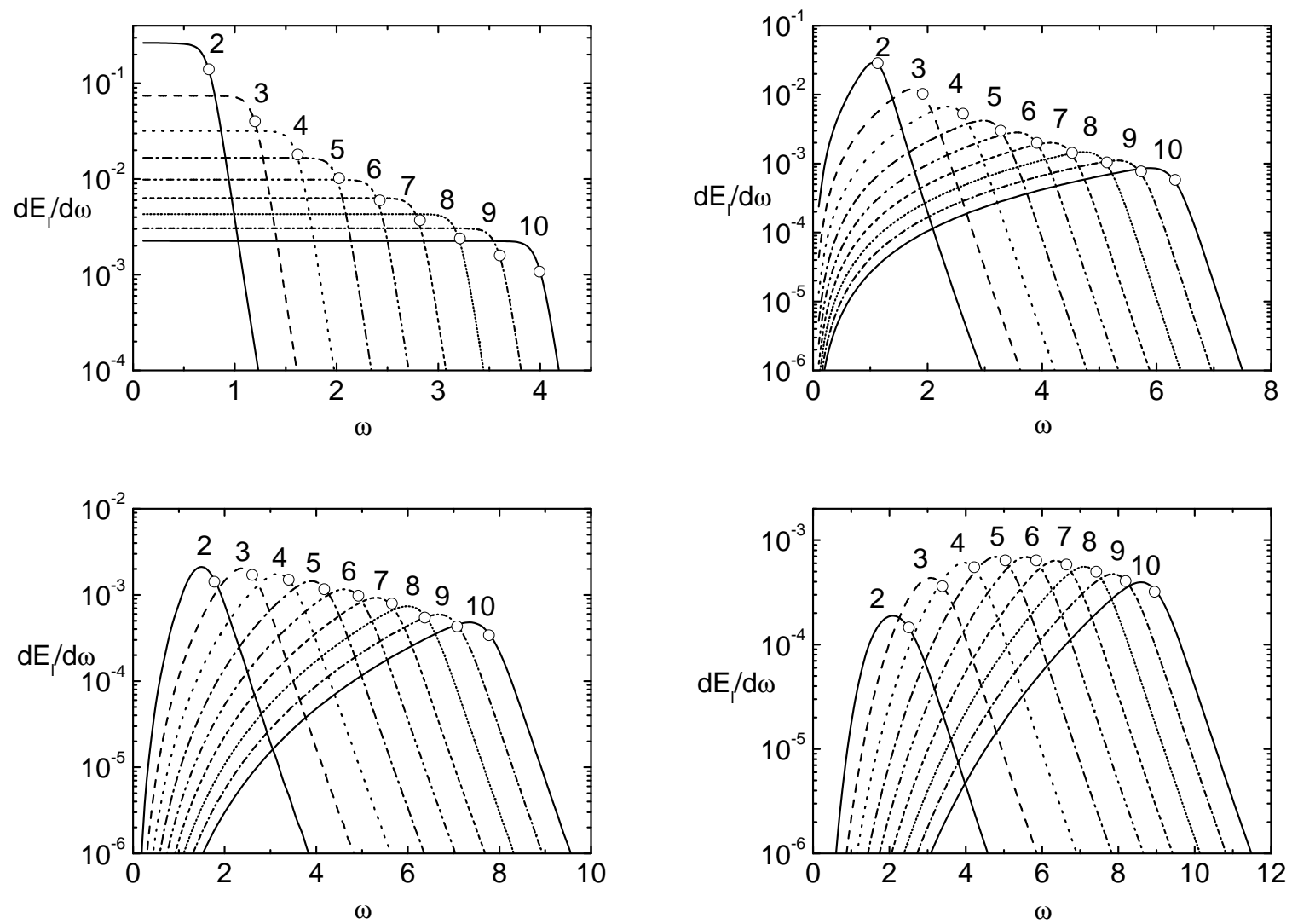

FIG. 1: Multipolar components of the energy spectra up to $l=10$ for $n=2, n=4$ (top left and top right panels), $n=6$ and $n=8$ (bottom left and bottom right panels) in units $r_{h}=1$. Open circles mark the real part of the fundamental scalar gravitational quasinormal frequency, $\omega_{l n}$, for the given $l$ and $n$.
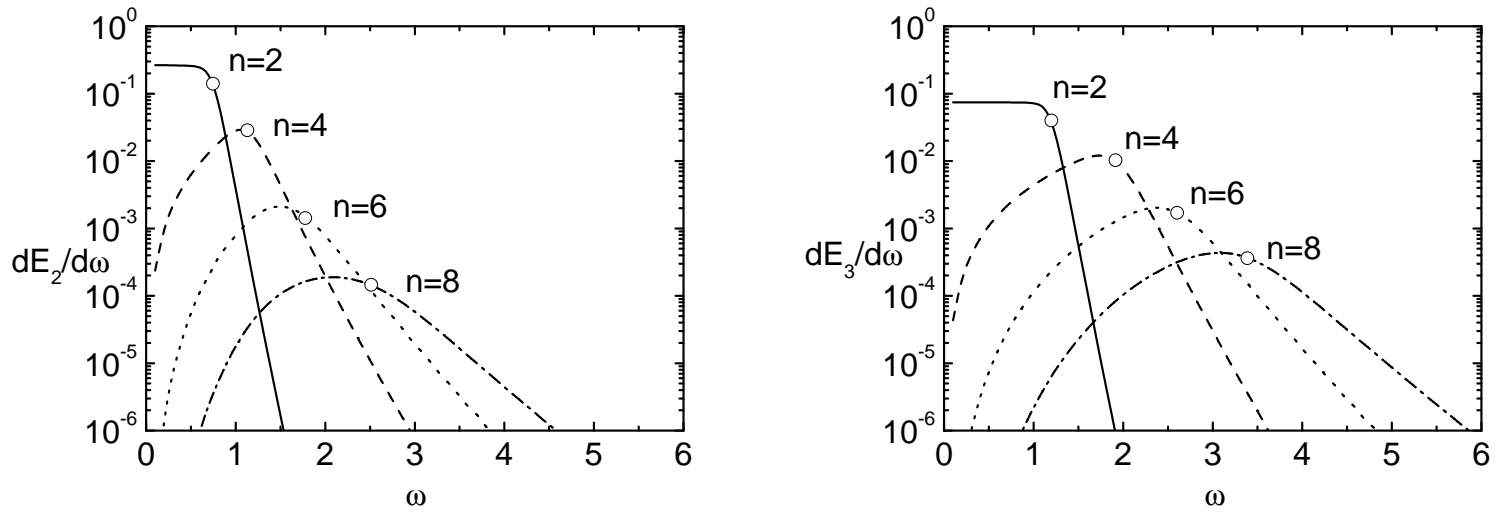

FIG. 2: Multipolar components of the energy spectra at fixed $l$ for different values of $n$ in units $r_{h}=1$. The left panel corresponds to $l=2$ and the right panel corresponds to $l=3$. Open circles mark the real part of the fundamental scalar gravitational quasinormal frequency, $\omega_{l n}$, for the given $l$ and $n$. 


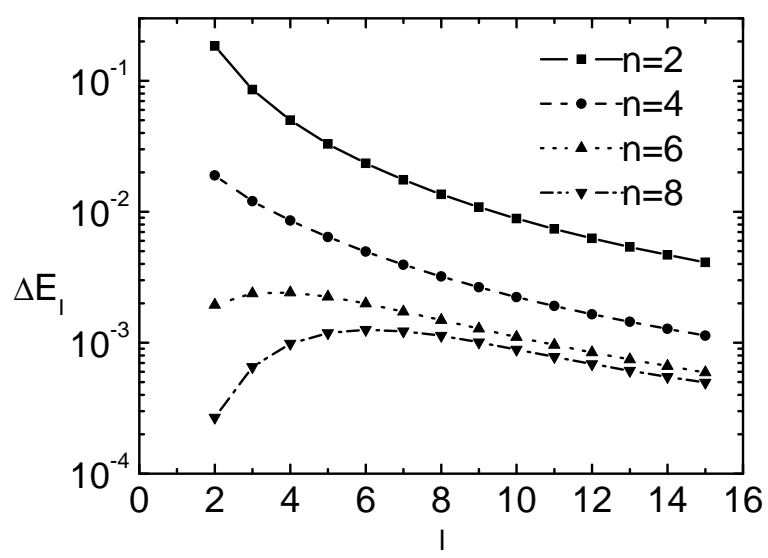

FIG. 3: The integrated energy $\Delta E_{l}$ as a function of $l$ for different values of $n$. The dominant multipolar component is $l=2$ only for $n<6$; this is probably related to the appearance of a negative well in the scalar potentials for $l=2$ and $n>4$. The dominant multipole is $l=4$ (6) for $n=6$ (8) (see Table V).

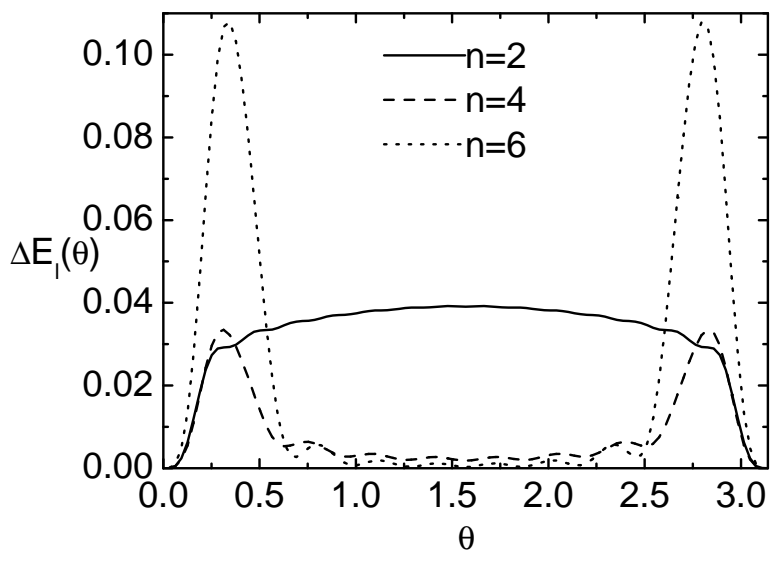

FIG. 4: Angular dependence of the radiation for $n=2,4$ and 6 , summing all multipoles up to $l=15$ in units $r_{h}=1$. The angular distribution for $n=8$ is not shown. The latter is even more peaked in a narrow region around $\theta=0$ since also the multipoles with $l>15$ contribute significantly to the radiation. 
TABLE I: QNMs for $n=2$. The first three quasinormal frequencies for scalar and vector perturbations are listed from left to right. The scalar modes and the vector modes are isospectral in four dimensions.

\begin{tabular}{|c|c|c|c|}
\hline $\mathrm{n}=2$ & \multicolumn{3}{|c|}{ Scalar and vector modes } \\
\hline $\mathrm{l}$ & $j=0$ & $j=1$ & $j=2$ \\
\hline 2 & $0.746-0.178 \mathrm{i}$ & $0.692-0.550 \mathrm{i}$ & $0.606-0.942 \mathrm{i}$ \\
3 & $1.199-0.185 \mathrm{i}$ & $1.165-0.563 \mathrm{i}$ & $1.106-0.953 \mathrm{i}$ \\
4 & $1.618-0.188 \mathrm{i}$ & $1.593-0.569 \mathrm{i}$ & $1.547-0.958 \mathrm{i}$ \\
5 & $2.025-0.190 \mathrm{i}$ & $2.004-0.572 \mathrm{i}$ & $1.967-0.960 \mathrm{i}$ \\
6 & $2.424-0.191 \mathrm{i}$ & $2.407-0.573 \mathrm{i}$ & $2.375-0.961 \mathrm{i}$ \\
7 & $2.819-0.191 \mathrm{i}$ & $2.805-0.574 \mathrm{i}$ & $2.777-0.961 \mathrm{i}$ \\
8 & $3.212-0.191 \mathrm{i}$ & $3.200-0.575 \mathrm{i}$ & $3.175-0.961 \mathrm{i}$ \\
9 & $3.604-0.192 \mathrm{i}$ & $3.592-0.575 \mathrm{i}$ & $3.570-0.962 \mathrm{i}$ \\
10 & $3.994-0.192 \mathrm{i}$ & $3.983-0.576 \mathrm{i}$ & $3.963-0.962 \mathrm{i}$ \\
11 & $4.383-0.192 \mathrm{i}$ & $4.373-0.576 \mathrm{i}$ & $4.355-0.962 \mathrm{i}$ \\
12 & $4.771-0.192 \mathrm{i}$ & $4.762-0.576 \mathrm{i}$ & $4.745-0.962 \mathrm{i}$ \\
13 & $5.159-0.192 \mathrm{i}$ & $5.151-0.576 \mathrm{i}$ & $5.135-0.962 \mathrm{i}$ \\
14 & $5.546-0.192 \mathrm{i}$ & $5.539-0.577 \mathrm{i}$ & $5.524-0.962 \mathrm{i}$ \\
15 & $5.934-0.192 \mathrm{i}$ & $5.927-0.577 \mathrm{i}$ & $5.913-0.962 \mathrm{i}$ \\
\hline
\end{tabular}

TABLE II: QNMs for $n=4$. The first three quasinormal frequencies for scalar, vector, and tensor perturbations are listed from left to right.

\begin{tabular}{|c|c|c|c|c|c|c|c|c|c|}
\hline $\mathrm{n}=4$ & & Scalar mor & & & Vec & & & de & \\
\hline 1 & $j=0$ & $j=1$ & $j=2$ & $j=0$ & $j=1$ & $j=2$ & $j=0$ & $j=1$ & $j=2$ \\
\hline 2 & $1.131-0.386 \mathrm{i}$ & $0.922-1.186 \mathrm{i}$ & $0.537-2.053 \mathrm{i}$ & $1.543-0.476 \mathrm{i}$ & $1.279-1.482 \mathrm{i}$ & $0.825-2.583 \mathrm{i}$ & $2.004-0.503 \mathrm{i}$ & $1.764-1.568 \mathrm{i}$ & $1.378-2.732 \mathrm{i}$ \\
\hline 3 & 1.91 & $1.715-1.217 \mathrm{i}$ & $1.336-2.103 \mathrm{i}$ & 2.191 & 1.988 & $1.625-2.492 \mathrm{i}$ & 2.576 & $2.393-1.531 \mathrm{i}$ & $2.632 \mathrm{i}$ \\
\hline 4 & $2.622-0.438 \mathrm{i}$ & $2.476-1.331 \mathrm{i}$ & $2.208-2.271 \mathrm{i}$ & 2.824-0.474i & $2.664-1.441 \mathrm{i}$ & $2.369-2.460 \mathrm{i}$ & $3.146-0.498 \mathrm{i}$ & $2.998-1.514 \mathrm{i}$ & $.580 \mathrm{i}$ \\
\hline 5 & $3.279-($ & $3.156-1$ & $2.924-2.347 \mathrm{i}$ & $3.441-0.478 \mathrm{i}$ & $3.310-1.447 \mathrm{i}$ & $3.063-2.453 \mathrm{i}$ & $3.716-0.497 \mathrm{i}$ & $3.592-1.504 \mathrm{i}$ & $.549 \mathrm{i}$ \\
\hline 6 & 301 & 3.80 & 3.598 & 4.04 & 3.93 & 3.72 & $496 \mathrm{i}$ & 4.17 & $.530 \mathrm{i}$ \\
\hline 7 & 4.527 & $429 \mathrm{i}$ & 4.249 & 4.644 & 4.5 & $4.360-2.456 \mathrm{i}$ & $496 \mathrm{i}$ & $4.762-1.495 \mathrm{i}$ & $2.517 \mathrm{i}$ \\
\hline 8 & 5.133 & $141 \mathrm{i}$ & $4.883-2$ & 5.236 & 5.150 & $4.983-2.458 \mathrm{i}$ & 5.42 & $5.342-1.492 \mathrm{i}$ & $5.178-2.508 \mathrm{i}$ \\
\hline 9 & 5.73 & $49 \mathrm{i}$ & $432 \mathrm{i}$ & 5.82 & 5.74 & $60 \mathrm{i}$ & $95 \mathrm{i}$ & 5.92 & $2.501 \mathrm{i}$ \\
\hline 10 & 6.326 & 6.25 & 6.118 & 6.409 & 6.33 & 6.20 & 6.56 & 6.49 & $6.361-2.496 \mathrm{i}$ \\
\hline 11 & 6.916 & 6.85 & 6.725 & 6.992 & 6.92 & 6.80 & 7.13 & 7.07 & $.492 \mathrm{i}$ \\
\hline 12 & 7.504 & 7.44 & $7.326-2.448 \mathrm{i}$ & 7.574 & 7.51 & & & 7.64 & 7.53 \\
\hline 13 & 8.088 & 8.03 & 7.923 & 8.15 & 8.09 & 7.988 & 8.27 & 8.22 & 8.11 \\
\hline 14 & 8.671 & & $8.517-2.454 \mathrm{i}$ & & 8.68 & & 8.8 & 8.79 & 8.69 \\
\hline 15 & $9.253-0.489 \mathrm{i}$ & $9.204-1.469 \mathrm{i}$ & $9.108-2.456 \mathrm{i}$ & $9.309-0.491 \mathrm{i}$ & $9.261-1.475 \mathrm{i}$ & $9.164-2.466 \mathrm{i}$ & $9.420-0.495 \mathrm{i}$ & $9.371-1.485 \mathrm{i}$ & $9.275-2.483$ \\
\hline
\end{tabular}


TABLE III: QNMs for $n=6$. The first three quasinormal frequencies for scalar, vector, and tensor perturbations are listed from left to right. The numbers in italic indicate that the potential at the given $l$ is not everywhere positive definite. The square brackets indicate that the potential has two scattering peaks.

\begin{tabular}{|c|c|c|c|c|c|c|c|c|c|}
\hline $\mathrm{n}=4$ & & Scalar modes & & & Vector modes & & & Tensor modes & \\
\hline$\overline{1}$ & $j=0$ & $j=1$ & $j=2$ & $j=0$ & $j=1$ & $j=2$ & $j=0$ & $j=1$ & $j=2$ \\
\hline 2 & [1.7778-0.571i] & {$[1.289-1.770 \mathrm{i}]$} & 395-3.201i] & $388-0.720 \mathrm{i}$ & $1.831-2.237_{1}$ & $0.825-4.001 \mathrm{i}$ & $2.956-0.751 \mathrm{i}$ & $2.365-2.357 \mathrm{i}$ & $339-4.245$ \\
\hline 3 & $2.604-0.628 \mathrm{i}$ & $2.198-1.916 \mathrm{i}$ & $.403-3.355 \mathrm{i}$ & $3.102-0.715 \mathrm{i}$ & $2.660-2.191 \mathrm{i}$ & $1.814-3.833 \mathrm{i}$ & $3.623-0.747 \mathrm{i}$ & $3.181-2.294 \mathrm{i}$ & $.351-4.012 \mathrm{i}$ \\
\hline 4 & $3.401-0.645 \mathrm{i}$ & $3.050-1.958 \mathrm{i}$ & $2.346-3.375 \mathrm{i}$ & $3.815-0.712 \mathrm{i}$ & $3.450-2.165 \mathrm{i}$ & $2.730-3.731 \mathrm{i}$ & $4.282-0.744 \mathrm{i}$ & $3.926-2.264 \mathrm{i}$ & $3.235-3.895 \mathrm{i}$ \\
\hline 5 & $4.174-0.660 \mathrm{i}$ & $3.875-1$. & $3.270-$ & $4.522-0$ & $4.213-2.156 \mathrm{i}$ & $3.595-3.678 \mathrm{i}$ & $4.940-0.741 \mathrm{i}$ & $4.640-2.247 \mathrm{i}$ & 4.047 \\
\hline 6 & $4.923-0.675 \mathrm{i}$ & $4.665-2.037 \mathrm{i}$ & $4.144-3.449 \mathrm{i}$ & $5.222-0.714 \mathrm{i}$ & $4.954-2.156 \mathrm{i}$ & $4.418-3.654 \mathrm{i}$ & $5.598-0.740 \mathrm{i}$ & $5.337-2.236 \mathrm{i}$ & $4.818-3.789$ \\
\hline 7 & $5.653-0$ & $5.425-2$ & $4.967-3$ & $716 \mathrm{i}$ & $5.679-2$ & 5.207- & 6.255 & 6.024 & 5.563 \\
\hline 8 & $6.369-0$ & $6.164-2$. & 5.753-3. & $6.602-0$ & $6.392-2$ & $5.969-3.643 \mathrm{i}$ & 6.913- & $6.705-$ & 6.290 \\
\hline 9 & $7.075-0.702 \mathrm{i}$ & \begin{tabular}{|l} 
6.888-2. \\
.
\end{tabular} & 6.515-3 & $7.285-0.721 \mathrm{i}$ & 7.094-2.169i & $6.712-3.644 \mathrm{i}$ & $7.570-0$ & 7.382 & $7.004-3.732 \mathrm{i}$ \\
\hline 10 & $7.772-0.707 \mathrm{i}$ & $7.602-2.128 \mathrm{i}$ & 7.259-3.570i & $7.964-0.722 \mathrm{i}$ & $7.790-2.173 \mathrm{i}$ & 7.441-3.646i & $8.228-0.737 \mathrm{i}$ & $8.055-2.218 \mathrm{i}$ & $7.709-3.722 \mathrm{i}$ \\
\hline 11 & $8.464-0.711 \mathrm{i}$ & 8.306-2.139i & 7.989-3.585i & $8.640-0.724 \mathrm{i}$ & $8.480-2.177 \mathrm{i}$ & $8.158-3.648 \mathrm{i}$ & $8.885-0.737 \mathrm{i}$ & $8.726-2.216 \mathrm{i}$ & $8.406-3.71$ \\
\hline 12 & 9.1 & 9. & 8.1 & bi & 9 & 8.867 & 9.543 & $9.395-$ & 9.098 \\
\hline 13 & $9.834-$ & $9.697-2$ & 9.421- & $9.986-$ & $9.847-$ & 9.569 & 10.200 & $10.062-$ & 9.785 \\
\hline 14 & $10.51-0.720 \mathrm{i}$ & $10.39-2.162 \mathrm{i}$ & $10.13-3.616 \mathrm{i}$ & $10.66-0.727 \mathrm{i}$ & $10.53-2.185 \mathrm{i}$ & $10.27-3.655 \mathrm{i}$ & $10.86-0.737 \mathrm{i}$ & $10.73-2.213 \mathrm{i}$ & $10.47-3.701$ \\
\hline 15 & $11.19-0.721 \mathrm{i}$ & $11.07-2.167 \mathrm{i}$ & $10.83-3.622 \mathrm{i}$ & $11.32-0.728 \mathrm{i}$ & $11.20-2.187 \mathrm{i}$ & $10.96-3.657 \mathrm{i}$ & $11.52-0.736 \mathrm{i}$ & $11.39-2.212 \mathrm{i}$ & $11.15-3.698$ \\
\hline
\end{tabular}

TABLE IV: QNMs for $n=8$. The first three quasinormal frequencies for scalar, vector, and tensor perturbations are listed from left to right. The numbers in italic indicate that the potential at the given $l$ is not everywhere positive definite. The square brackets indicate that the potential has two scattering peaks.

\begin{tabular}{|c|c|c|c|c|c|c|c|c|c|}
\hline $\mathrm{n}=4$ & \multicolumn{3}{|c|}{ Scalar modes } & \multicolumn{3}{|c|}{ Vector modes } & \multicolumn{3}{|c|}{ Tensor modes } \\
\hline 1 & $j=0$ & $j=1$ & $j=2$ & $j=0$ & $j=1$ & $j=2$ & $j=0$ & $j=1$ & $j=2$ \\
\hline $1=2$ & [2.513-0.744ii] & [1.686-2.299i] & {$[0.159-4.345 \mathrm{i}]$} & $3.261-0.924 \mathrm{i}$ & $2.335-2.851 \mathrm{i}$ & $0.598-5.287$ & $3.886-0.959 \mathrm{i}$ & $2.765-2.988 \mathrm{i}$ & $0.706-5.720 \mathrm{i}$ \\
\hline $1=3$ & [3.388-0.812i] & {$[2.696-2.461 \mathrm{i}]$} & [1.277-4.431i] & $4.017-0.923 \mathrm{i}$ & 3.269-2.804i & $1.747-5.016 \mathrm{i}$ & $4.618-0.959 \mathrm{i}$ & $3.806-2.917 \mathrm{i}$ & $2.141-5.241 \mathrm{i}$ \\
\hline$l=4$ & $4.223-0.841 \mathrm{i}$ & $3.631-2.532 \mathrm{i}$ & $2.367-4.420 \mathrm{i}$ & $4.775-0.920 \mathrm{i}$ & $4.147-2.777 \mathrm{i}$ & $2.824-4.840 \mathrm{i}$ & $5.336-0.955 \mathrm{i}$ & $4.691-2.885 \mathrm{i}$ & $3.331-5.018 \mathrm{i}$ \\
\hline$l=5$ & $5.042-0.855 \mathrm{i}$ & $4.524-2.568 \mathrm{i}$ & $3.407-4.401 \mathrm{i}$ & $5.531-0.918 \mathrm{i}$ & $4.991-2.762 \mathrm{i}$ & $3.840-4.734 \mathrm{i}$ & $6.049-0.951 \mathrm{i}$ & $5.507-2.866 \mathrm{i}$ & $4.360-4.904 \mathrm{i}$ \\
\hline $1=6$ & $5.848-0.865 \mathrm{i}$ & $5.390-2.595 \mathrm{i}$ & $4.403-4.399 \mathrm{i}$ & $6.283-0.917 \mathrm{i}$ & $5.810-2.757 \mathrm{i}$ & $4.802-4.676 \mathrm{i}$ & $6.761-0.949 \mathrm{i}$ & $6.291-2.854 \mathrm{i}$ & $5.297-4.838 \mathrm{i}$ \\
\hline $1=7$ & $6.640-0.874 \mathrm{i}$ & $6.231-2.622 \mathrm{i}$ & $5.357-4.415 \mathrm{i}$ & $7.030-0.918 \mathrm{i}$ & $6.610-2.757 \mathrm{i}$ & $5.719-4.646 \mathrm{i}$ & $7.473-0.947 \mathrm{i}$ & $7.056-2.846 \mathrm{i}$ & $6.178-4.798 \mathrm{i}$ \\
\hline $1=8$ & $7.420-0.883 \mathrm{i}$ & $7.052-2.647 \mathrm{i}$ & $6.270-4.441 \mathrm{i}$ & $7.774-0.920 \mathrm{i}$ & 7.396-2.759i & $6.599-4.634 \mathrm{i}$ & $8.184-0.946 \mathrm{i}$ & $7.808-2.841 \mathrm{i}$ & 7.022-4.772i \\
\hline $1=9$ & $8.191-0.890 \mathrm{i}$ & $7.855-2.669 \mathrm{i}$ & $7.149-4.469 \mathrm{i}$ & $8.514-0.921 \mathrm{i}$ & $8.167-2.764 \mathrm{i}$ & $7.450-4.630 \mathrm{i}$ & $8.895-0.945 \mathrm{i}$ & $8.553-2.837 \mathrm{i}$ & 7.841-4.755i \\
\hline $1=10$ & $8.953-0.896 \mathrm{i}$ & $8.645-2.688 \mathrm{i}$ & $7.999-4.495 \mathrm{i}$ & $9.250-0.923 \mathrm{i}$ & $8.935-2.768 \mathrm{i}$ & $8.278-4.630 \mathrm{i}$ & $9.606-0.944 \mathrm{i}$ & $9.292-2.834 \mathrm{i}$ & $8.640-4.743 \mathrm{i}$ \\
\hline $1=11$ & $9.709-0.902 \mathrm{i}$ & $9.423-2.705 \mathrm{i}$ & $8.829-4.519 \mathrm{i}$ & $9.984-0.924 \mathrm{i}$ & 9.692-2.773i & $9.088-4.633 \mathrm{i}$ & $10.32-0.944 \mathrm{i}$ & $10.03-2.832 \mathrm{i}$ & $9.426-4.735 \mathrm{i}$ \\
\hline$l=12$ & $10.46-0.906 \mathrm{i}$ & $10.19-2.718 \mathrm{i}$ & $9.641-4.539 \mathrm{i}$ & $10.71-0.926 \mathrm{i}$ & $10.44-2.777 \mathrm{i}$ & 9.884-4.637i & $11.03-0.943 \mathrm{i}$ & $10.76-2.831 \mathrm{i}$ & $10.20-4.728 \mathrm{i}$ \\
\hline $1=13$ & $11.20-0.910 \mathrm{i}$ & $10.95-2.730 \mathrm{i}$ & $10.44-4.556 \mathrm{i}$ & $11.44-0.927 \mathrm{i}$ & $11.19-2.781 \mathrm{i}$ & $10.67-4.642 \mathrm{i}$ & $11.74-0.943 \mathrm{i}$ & $11.49-2.829 \mathrm{i}$ & $10.97-4.724 \mathrm{i}$ \\
\hline$l=14$ & $11.95-0.914 \mathrm{i}$ & $11.71-2.740 \mathrm{i}$ & $11.23-4.571 \mathrm{i}$ & $12.17-0.928 \mathrm{i}$ & $11.93-2.785 \mathrm{i}$ & $11.44-4.646 \mathrm{i}$ & $12.45-0.943 \mathrm{i}$ & $12.21-2.828 \mathrm{i}$ & $11.73-4.720 \mathrm{i}$ \\
\hline $1=15$ & $12.68-0.916 \mathrm{i}$ & $12.46-2.748 \mathrm{i}$ & $12.01-4.584 \mathrm{i}$ & $12.90-0.929 \mathrm{i}$ & $12.67-2.788 \mathrm{i}$ & $12.21-4.650 \mathrm{i}$ & $13.16-0.943 \mathrm{i}$ & $12.94-2.828 \mathrm{i}$ & $12.48-4.718 \mathrm{i}$ \\
\hline
\end{tabular}


TABLE V: Multipolar contributions to the total energy for different $n$ in units $r_{h}=1$.

\begin{tabular}{|c|c|c|c|c|}
\hline$l$ & $n=2$ & $n=4$ & $n=6$ & $n=8$ \\
\hline 2 & 0.1845 & $0.189 \mathrm{e}-1$ & $0.194 \mathrm{e}-2$ & $0.269 \mathrm{e}-3$ \\
3 & 0.0855 & $0.120 \mathrm{e}-1$ & $0.238 \mathrm{e}-2$ & $0.653 \mathrm{e}-3$ \\
4 & 0.0500 & $0.086 \mathrm{e}-1$ & $0.241 \mathrm{e}-2$ & $0.983 \mathrm{e}-3$ \\
5 & 0.0329 & $0.064 \mathrm{e}-1$ & $0.224 \mathrm{e}-2$ & $1.187 \mathrm{e}-3$ \\
6 & 0.0234 & $0.050 \mathrm{e}-1$ & $0.199 \mathrm{e}-2$ & $1.258 \mathrm{e}-3$ \\
7 & 0.0175 & $0.039 \mathrm{e}-1$ & $0.172 \mathrm{e}-2$ & $1.225 \mathrm{e}-3$ \\
8 & 0.0136 & $0.032 \mathrm{e}-1$ & $0.149 \mathrm{e}-2$ & $1.130 \mathrm{e}-3$ \\
9 & 0.0109 & $0.027 \mathrm{e}-1$ & $0.128 \mathrm{e}-2$ & $1.009 \mathrm{e}-3$ \\
10 & 0.0089 & $0.022 \mathrm{e}-1$ & $0.111 \mathrm{e}-2$ & $0.888 \mathrm{e}-3$ \\
11 & 0.0074 & $0.019 \mathrm{e}-1$ & $0.096 \mathrm{e}-2$ & $0.780 \mathrm{e}-3$ \\
12 & 0.0063 & $0.016 \mathrm{e}-1$ & $0.084 \mathrm{e}-2$ & $0.688 \mathrm{e}-3$ \\
13 & 0.0054 & $0.014 \mathrm{e}-1$ & $0.075 \mathrm{e}-2$ & $0.612 \mathrm{e}-3$ \\
14 & 0.0047 & $0.013 \mathrm{e}-1$ & $0.066 \mathrm{e}-2$ & $0.549 \mathrm{e}-3$ \\
15 & 0.0041 & $0.011 \mathrm{e}-1$ & $0.059 \mathrm{e}-2$ & $0.497 \mathrm{e}-3$ \\
\hline
\end{tabular}

TABLE VI: Total energy for different spacetime dimensions. From left to right, the columns give the spacetime dimension $D=n+2$, the factor $n \mathcal{A}_{n} / 16 \pi$, the total energy $E_{\text {tot }}^{(D)}$ in units $r_{h}=1$, the rescaled total energy $\mathcal{E}_{\text {tot }}^{(D)}=n \mathcal{A}_{n} E_{\text {tot }}^{(D)} / 16 \pi$ and the gravitational energy loss (see text).

\begin{tabular}{|c|c|c|c|c|}
\hline$D$ & $n \mathcal{A}_{n} / 16 \pi$ & $E_{\text {tot }}^{(D)}$ & $\mathcal{E}_{\text {tot }}^{(D)}$ & Energy loss \\
\hline 4 & $1 / 2$ & 0.52 & 0.26 & $13 \%$ \\
6 & $2 \pi / 3$ & 0.095 & 0.20 & $10 \%$ \\
8 & $2 \pi^{2} / 5$ & 0.034 & 0.13 & $7 \%$ \\
10 & $16 \pi^{3} / 105$ & 0.032 & 0.15 & $8 \%$ \\
\hline
\end{tabular}

